\title{
Las nuevas normas españolas para cemento*
}

\section{N T R O D U C C I O N}

\section{SEÑORES:}

Es para mi un alto honor, que no es la primera vez que recibo de la fraternal amistad de ustedes los hermanos argentinos, el haber sido invitado por el Instituto Nacional de Tecnología Industrial (INTI) con la colaboración del Instituto del Cemento Portland Argentino (ICPA), para departir sobre temas que profesionalmente nos interesan y preocupan.

$\mathrm{Al}$ aceptar con todo agrado tan amable y cortés invitación, quiero agradecerla en lo mucho que significa, y quiero corresponder a ella ofreciéndoles -mejor sería decir haciéndoles soportar- una exposición acerca de las Nuevas Normas Españolas para Cemento. Se puede pensar que es una forma rara de agradecer, pero en el buen deseo va puesto el corazón.

El haber elegido este tema, o por mejor decir, este título para mi exposición, no ha sido tanto por hablarles de normas españolas, cuanto por que ello sirva de base para comentar y, si a mano viene, incluso criticar, en coloquio amplio, las tendencias y aspectos particulares de las normas para cemento, en general.

Por ello y para ello, a lo largo de esta exposición trataré de hacer a veces de abogado del diablo, con objeto de suscitar cuestiones y hacer destacar matices que les den a ustedes pie para intervenir en el coloquio que se pueda entablar.

\section{HISTORIA}

Hasta 1960 estuvo vigente en España el Pliego de Cementos de 1930 (1), a la sazón anticuado a todas luces. Se imponía, pues, actualizarlo, a tenor de las normas extranjeras más al día. Pero semejante actualización, dadas las circunstancias de desfase del Pliego Español de 1930 con respecto a dichas normas extranjeras, hubo de consistir más bien en la elaboración completa de unas nuevas normas para cemento, prácticamente partiendo de cero.

* Conferencia pronunciada en la Associaçao Brasileira de Cemento Portland (ABCP), de Sao Paulo, Brasil, en Mayo de 1976, y en el Instituto Nacional de Tecnología Industrial (INTI), de Buenos Aires, Argentina, en Junio de dicho año. 
Así surgió el Pliego de Cementos de 1960, cuyo texto fue recogido al año siguiente en la publicación PCCH-61 (Pliego General de Condiciones para la Recepción de Conglomerantes Hidráulicos en las Obras de Carácter Oficial) de la serie "Normas y Manuales" del IETCC (2).

El PCCH-61 constaba de un documento en el que se daba una serie de definiciones y designaciones escuetas de los cementos en sí, sin otra inclusión que la de las definiciones, muy sucintas, de clínker, escoria siderúrgica y puzolana. En él se daban también dos cuadros de prescripciones, unas relativas a la composición química, y otras referentes a las características físicas y mecánicas de los cementos, así como un apartado concerniente a prescripciones especiales: una de expansión en autoclave para cementos llamados "de alta estabilidad", otra para cementos de bajo contenido de álcalis, y otra para cementos de bajo calor de hidratación. Finalmente, el PCCH-61 constaba de otros dos documentos: uno relativo a toma de muestras y otro dedicado a métodos de ensayo.

En 1964 se revisó el Pliego de 1960, siendo recogido el nuevo texto revisado en la publicación PCCH-64 de la ya mencionada serie "Normas y Manuales" del IETCC, con el mismo título (3).

Las diferencias sustanciales, aparte de las de ordenación, que supuso el paso del PCCH-61 al PCCH-64, pueden describirse así:

\section{A) En cuanto a tipos y categorías resistentes de los cementos}

a) supresión de la categoría inferior de cementos, 150, en los tipos portland, siderúrgicos y puzolánicos;

b) introducción de una categoría superior, 450, en los cementos de tipo portland;

c) introducción de dos nuevas categorías resistentes superiores, 250 y 350, en los cementos portland resistentes a aguas selenitosas (yesos);

d) introducción de una nueva categoría resistente, 350, en los cementos siderúrgicos y de horno alto;

e) introducción de un nuevo tipo de cemento: el DE ADICION, en el que se incluyó el cemento siderúrgico-clínker SC-150 del PCCH-61, y el nuevo cemento C-150, una especie de cemento de albañilería, con hasta 35 por ciento de adiciones inertes totales;

f) supresión de los cementos llamados "de alta estabilidad" (expansión y contracción en autoclave menores de 0,7 y 0,3 por ciento respectivamente).

\section{B) En cuanto a prescripciones y a métodos de ensayo}

a) supresión del método para la determinación de la granulometría del cemento con separador de aire;

b) introducción del método del permeabilímetro de BLAINE para determinar la superficie específica de los cementos, y prescripción de valores máximos y mínimos para los distintos tipos, clases y categorías de cementos (aparte de la prescripción relativa al residuo sobre tamiz de 4.900 mallas $/ \mathrm{cm}^{2}$, del $\mathrm{PCCH}-61$, la cual se mantuvo moidificada);

c) supresión del ensayo de expansión de los cementos mediante galletas de pasta pura; 
d) extensión del método del autoclave para medir la expansión de los cementos, y prescripción de un valor máximo de 1 por ciento para los portland, siderúrgicos y puzolánicos ;

e) supresión de la "alta estabilidad" (expansión y contracción en autoclave menores de 0,7 y $0,3 \%$ respectivamente), como prescripción especial para los cementos de "alta estabilidad", asimismo suprimidos;

f) introducción de métodos de análisis químico para los cementos DE ADICION;

g) introducción del método de fotometría de llama para la determinación de álcalis en cementos (además del método químico del PCCH-61, que se mantuvo).

Aparte de estas diferencias, y por lo que se refiere a las prescripciones o especificaciones de carácter químico, se igualaron por arriba algunos límites máximos admisibles de óxido magnésico, trióxido de azufre, pérdida al fuego y aluminato tricálcico, en algunas clases y categorías, nuevas o viejas, de los cementos de tipo portland. En este sentido el cambio PCCH-61 al PCCH-64 fue, en general, lógico y liberalizador.

Otra diferencia del PCCH-64 respecto del PCCH-61 es la introducción de notaciones marginales explicativas o aclaratorias en el primero de los pliegos citados.

Como es de apreciar, también, el paso del PCCH-61 al PCCH-64 supuso mejoras en las calidades resistentes de los cementos (eliminación de las categorías más bajas y creación de otras más altas).

Supuso también una preocupación, no muy bien fundada entonces, por la expansión (adopción del método del autoclave), así como por la retracción (especificación de máximos de superficie específica BLAINE), de los cementos.

Por la expansión porque, ante las nuevas categorías de cementos más resistentes se preveían, tal vez, mayores grados de saturación en los crudos y más cal libre en los clínkeres y en los cementos, así como más aluminatos para lograr mayores resistencias a plazos muy cortos.

Pero, en cambio, no se previó la influencia que en la invalidación de los resultados del ensayo del autoclave pudiera tener, sobre todo en algunos casos, el hecho al parecer trivial de que, tanto el PCCH-61 como el PCCH-64, denominaran "cementos portland comerciales" a los que, además de los componentes principales, clínker y piedra de yeso, contuviesen otras adiciones no nocivas en proporción inferior al 10 por ciento... Ni que decir tiene que, a partir de tal posiblidad, prácticamente todos los fabricantes del país hicieron "cemento portland comercial". Pero un 10 por ciento de adición activa (puzolana, ceniza volante o escoria siderúrgica), o incluso aparentemente inerte (marga), por el llamado y conocido "efecto silícico" que tantas veces he mencionado, hace estables en el autoclave a cementos que en la práctica pudieran ser expansivos. El mito de la infalibilidad y de la seguridad de los resultados del método de ensayo de expansión de cementos en autoclave, como tantos otros mitos, se viene así abajo.

Por la retracción, porque es cierto que ésta aumenta con la finura, y las nuevas categorías más resistentes de los cementos del $\mathrm{PCCH}-64$ tal vez exigiesen algo mayores finuras de molido. Pero no es menos cierto que, dados los avances en la maquinaria y en la técnica de la fabricación (en aquella fecha España ya había comenzado su despegue), el fabricante no precisaba de forzar las finuras en demasía para conseguir con amplio margen las resistencias mínimas de las categorías nuevas.

Por otra parte, la alternativa de introducir en las normas una especificación y un ensayo de retracción era -y sigue siendo- muy comprometida y problemática. En primer lugar, porque no existía - ni existe hoy, que yo sepa- en ninguna norma. En segundo lugar, porque ¿qué valor de especificación proponer y cómo justificarlo? En tercer lugar, por- 
que ¿qué método adoptar? El método francés del anillo de pasta pura, que a veces y por algunos se ha considerado como procedimiento de medir la retracción, no es tal. Lo que mide en realidad es la fisurabilidad de la pasta, uno de cuyos componentes - como la resistencia a la tracción y como el módulo de elasticidad- es la retracción.

En los dos últimos aspectos tratados, expansión y finura, el paso del PCCH-61 al PCCH-64 no fue precisamente, ni muy justificado, ni mucho menos liberalizador, al menos en la forma.

A partir de 1974, y por disposición gubernamental, se comenzó la revisión del PCCH-64 a cargo de una Comisión Interministerial y de un Grupo de Trabajo. En la revisión se tuvieron en cuenta los progresos en la técnica de la fabricación de cementos y la evolución de las normas de los distintos países en los últimos tiempos. La revisión concluyó en 1975, y el nuevo texto revisado se recoge en la publicación RC-75 (Recepción de Cementos-1975) de la Secretaría General Técnica del Ministerio de Obras Públicas.

El Pliego RC-75 consta de ocho capítulos dedicados respectivamente a: definiciones y denominaciones de los componentes de los cementos; definiciones, clasificación, denominaciones y designaciones de los cementos; características -especificaciones (físicas, mecánicas y químicas) - de los cementos; envasado e identificación; toma de muestras; métodos de ensayo; métodos para determinar las características químicas.

En relación con el PCCH-64 el Pliego RC-75 presenta notables diferencias. La primera afecta al nombre o título, ya que el actual del RC-75 es "Pliego de Prescripciones Técnicas Generales para la Recepción de Cementos", con el cual se da a entender que sus prescripciones se extienden a todo el ámbito nacional, de acuerdo con el Reglamento General de Contratación.

E1 resto de las diferencias se puede exponer así, tanto en cuanto al PCCH-64 como en cuanto al PCCH-61:

\section{I) Por lo que respecta a definiciones y denominaciones}

i) a diferencia del PCCH-61 y del PCCH-64 el Pliego RC-75 no sólo define el clínker, las puzolanas y las escorias (éstas con mayor detalle que aquéllos), sino que entra también a definir los restantes componentes de los clínkeres y de los cementos, tales como los crudos de cementos portland y aluminosos, los clínkeres de los cementos aluminosos y naturales, los reguladores de fraguado y las adiciones tanto activas como inertes.

\section{II) Por lo que afecta a los cementos en sí}

i) se introducen nuevos tipos y clases de cementos, como los portland con adiciones activas (escorias, puzolanas y cenizas volantes), los puzolánicos con cenizas volantes y los COMPUESTOS. Estos últimos vienen a sustituir en parte a los DE ADICION del PCCH-64;

ii) siguiendo la línea de la transición del PCCH-61 al PCCH-64, se suprime en varios tipos y clases de cemento la categoría resistente inferior, 250; 
iii) dentro de la misma línea se introduce una categoría resistente superior, 550, en los cementos portland y. portland con adiciones activas;

iv) análogamente se introducen categorías resistentes superiores, 450, en los cementos siderúrgicos y puzolánicos;

v) se suprimen los cementos siderúrgicos sobresulfatados o sulfosiderúrgicos y los siderúrgico-clínker que nunca se habían llegado a producir en España;

vi) se introducen los cementos puzolánicos a base de cenizas volantes;

vii) se sustituyen los cementos DE ADICION (más o menos de albañilería) por los CEMENTOS COMPUESTOS, suprimiendo de entre ellos el mencionado cemento siderúrgico-clínker y cambiando el C-150 del PCCH-64 por un nuevo C-200 (gris o blanco) ;

viii) se suprime el cemento "Zumaya", una especie de cemento natural marítimo, de fabricación restringida en una sola zona y localidad de la Península, el cual queda incluido como uno más en su grupo de cementos naturales.

ix) se crea un grupo de cementos con propiedades adicionales: resistencia inicial, resistencia al yeso y bajo calor de hidratación, dentro de los portland; así como con la condición de blancura, dentro de los portland y de los compuestos;

x) al cemento aluminoso se le eleva de forma notable la categoría resistente.

\section{III) Por lo que se refiere a prescripciones o especificaciones y a métodos de ensayo}

i) se suprime el método para la determinación de la supeficie específica BLAINE, así como las limitaciones máxima y mínima de dicha superficie en los cementos, dejando sólo la prescripción de finura por residuo máximo sobre tamiz de 4.900 mallas $/ \mathrm{cm}^{2}$ (igual para todos los cementos: 15 por ciento);

ii) se mantiene el método del autoclave para medir la expansión de los cementos portland solamente (con la misma limitación del 1 por ciento);

iii) se introduce el ensayo de las agujas Le Chatelier para determinar la expansión de los restantes cementos (excepto los naturales y los aluminosos);

iv) se autoriza la utilización de métodos rápidos o acelerados de análisis químico, por acuerdo entre partes, y sin carácter sustitutorio del método prescriptivo, único fidedigno en caso de controversia;

v) se da una ordenación distinta a los procedimientos analíticos, agrupando los relativos a una misma determinación, pero que son alternativos u optativos, o se aplican en distintos casos o a diferentes tipos de cementos (por ejemplo, los métodos para determinar residuo insoluble, óxido férrico, sílice, cal, etc.);

vi) se suprime el método químico para la determinación de álcalis en cementos y se mantiene en exclusiva el método fotométrico de llama;

vii) se sustituye el método de la glicerina para determinar la cal libre, por el método del etilenglicol;

viii) se introduce un método para medir la blancura de los cementos blancos y se establece una especificación relativa a la misma; 
ix) se suprime el ábaco para la determinación de la composición potencial de los cementos, de empleo incómodo y restringido, y se mantienen exclusivamente las fórmulas extendidas a los distintos casos posibles en función de la relación $\mathrm{Al}_{2} \mathrm{O}_{3} /$ $/ \mathrm{Fe}_{2} \mathrm{O}_{3}$ de los cementos;

$\mathrm{x}$ ) finalmente, el Pliego RC-75 es mucho más explícito y detallado que los PCCH-61 y PCCH-64 en cuanto al capítulo de toma de muestras, incluyendo otro relativo a envasado e identificación de los cementos.

Es de advertir que en determinados aspectos el Pliego RC-75 es digeramente más liberal que el PCCH-64, y en otros menos.

Las definiciones de los componentes y materias primas de los cementos, y aún de los clínkeres de los mismos se pueden interpretar como una oficiosidad innecesaria, o como información más propia de un manual o libro de texto, pues al fabricante no hay por qué decirle con qué y cómo tiene que hacer el cemento, ya que conoce su oficio; se le puede y se le debe exigir, eso sí, que el cemento que fabrique cumpla con una serie de especificaciones, que son precisamente las que figuran en las normas.

El Pliego RC-75 es algo más restrictivo que el PCCH-64 en algún caso, en cuanto a los límites tolerables de pérdida al fuego y de residuo insoluble. En cuanto al límite de trióxido de azufre adopta un criterio razonablemente ecléctico, a saber: en relación con el valor máximo de 4 por ciento impuesto por el PCCH-64 para todos los cementos, aumenta dicho valor a 4,5 en ciertos casos y lo reduce a 3,5 en otros. Lo primero, en los cementos portland de las categorías resistentes más altas, por razón de su mayor finura, y en los cementos siderúrgicos, por razón de su contenido de escorias; lo segundo, en el caso de los cementos puzolánicos, por razón de la sustitución de una buena parte de clínker por puzolana, no sensible al yeso.

Por otro lado, con relación al PCCH-64 el Pliego RC-75 se ha liberado del lastre del ensayo del autoclave para los cementos no portland (siderúrgicos y puzolánicos) y portland con adiciones activas (hasta 20 por ciento de escorias siderúrgicas, puzolanas o cenizas volantes), para los cuales no es operante en virtud del "efecto silícico". Asimismo se ha liberado del ensayo de determinación de superficie específica BLAINE y de la especifica"ción sobre máximos y mínimos de la misma.

Se ha liberado, sobre todo, del hecho insólito de la simultaneidad de dos especificaciones alternativas y optativas sobre finura del cemento: una dada en función de un residuo muy tolerante sobre tamiz de 4.900 mallas $/ \mathrm{cm}^{2}$; y otra en función de los susodichos máximos y mínimos de finura BLAINE. Por supuesto que al ser optativos tanto las dos especificaciones como los respectivos métodos para determinarlas según el PCCH-64, los fabricantes de cemento se atenían al residuo sobre tamiz, con lo cual los máximos y los mínimos BLAINE quedaban inoperantes. La supresión de éstos -feliz por tales circunstancias- en el Pliego RC-75, no invalida al método de BLAINE ni a la superficie específica como auxiliar indicativo de gran utilidad para control en fábrica.

\section{ACTUALIDAD}

En el momento presente el Pliego RC-75, visto en su conjunto, presenta el aspecto general y los particulares de los cuadros que se muestran.

Comentarios críticos sobre el mismo pueden ser los siguientes:

1) Si se tienen en cuenta todos los tipos, clases y categorías de cementos, resultan 33 cementos diferentes, por uno u otro concepto. Son muchos cementos. Máxime cuando algunos de ellos probablemente sólo pueden existir sobre el papel, y cuando otros 
están afectados por especificaciones que no son correlativas. Por ejemplo, al cemento P-350-ARI se le exige, por el hecho de ser P-350, una resistencia mínima de 250 $\mathrm{kg} / \mathrm{cm}^{2}$ a 7 días; mientras que por el hecho de ser ARI se le exige dicha resistencia mínima a 2 días. Es evidente que con esta condición sobra, por superflua, la anterior. Sobra incluso la otra condición del P-350, esto es, la de dar una resistencia mínima de $350 \mathrm{~kg} / \mathrm{cm}^{2}$ a 28 días, pues con 250 a 2 días es seguro que la cumple, aunque no se especifique. Dicho de otro modo, los cementos ARI con la condición de resistencia mínima de $250 \mathrm{~kg} / \mathrm{cm}^{2}$ a 2 días siempre tendrían categoría superior a la de un P-350 corriente. Por lo tanto, lo lógico sería considerar sólo dos cementos de altas resistencias iniciales: el P-450-ARI y el P-550-ARI. Y más sensato aún sería considerar uno: el que diera un mínimo de $250 \mathrm{~kg} / \mathrm{cm}^{2}$ a 2 días, sin ninguna otra especificación de resistencia a otras edades, en la seguridad de que tal cemento daría a 28 días resistencias comprendidas entre 450 y $550 \mathrm{~kg} / \mathrm{cm}^{2}$, y probablemente superiores en algún caso. Tales cementos se pueden considerar como de alta resistencia a toda edad. Pero este aspecto bien merece un comentario.

En el compendio de normas del CEMBUREAU (5) se consideran sinónimas las expresiones "cemento portland de alta resistencia inicial, o a corto plazo, o a corta o temprana edad...", y "cemento portland de endurecimiento rápido".

Aparte de ellas se tiene en cuenta también, como distinta, la expresión "cemento portland de alta resistencia" (se entiende que, si no "inicial", sí "final"). No existe una clara y discriminatoria definición cuantitativa de cementos portland de alta resistencia (a secas, pero debiéndose entender que final o a largo plazo -28 días-), en relación con los cementos portland de endurecimiento rápido o de alta resistencia ínicial a 1, 2 ó 3 días. Hasta tal punto, que no siempre se utilizan las dos expresiones con $\in 1$ mismo valor, en cada caso, por parte de países diferentes. Por el contrario, algunos las utilizan (entre sí) de forma cruzada. Si se considera el caso de aquellos países cuyas normas incluyen cementos de alta resistencia y cementos de endurecimiento rápido (a fin de poder comparar sobre una misma base en todo caso), se observan los siguientes hechos:

a) que a los cementos de endurecimiento rápido a veces se les exige, en relación con los "correspondientes" de alta resistencia:

i) resistencias mayores a todas las (mismas) edades consideradas (Bélgica, Turquía);

ii) la misma resistencia final, pero resistencias mayores a edades intermedias y sobre todo, más tempranas (Checoslovaquia, Alemania Federal);

iii) las mismas resistencias finales y a edades tempranas, sin especificar en ningún caso resistencias a edades intermedias (Alemania Federal);

iv) las mismas resistencias a edades tempranas, sin especificar resistencias finales ni a edades intermedias. En tales casos la denominación de los cementos suele ser híbrida: "cementos portland de rápida resistencia alta" —o de alta resistencia conseguida desde las primerísimas edades- (Méjico);

v) raramente ( $\mathrm{y}$ en contraposición a i), resistencias menores a todas las edades (pero no las mismas edades) consideradas (Polonia);

b) que, bien sea dentro de los cementos de endurecimiento rápido, o bien dentro de los de alta resistencia, hay países que establecen clases y categorías (República Democrática Alemana, República Federal de Alemania, Hungría, Polonia y Grecia*, entre otros.

* Es curioso consignar que en el caso de Crecia los cementos de endurecimiento rápido o de alta resistencia inicial, pueden contener hasta un $10 \%$ de tierra de Santorín (puzolana natural). 
c) que la resultante de a) y b) es que, en general, a los cementos de endurecimiento rápido se les exige, en relación con los de alta resistencia, y en todos los países que especifican sobre ambas clases de cemento, mayores (en algún caso iguales) resistencias a plazos cortos - uno o dos días-, intermedios - tres o siete días-o largos --veintiocho días-. Esto es congruente con lo expuesto por el que habla, a propósito de que cementos ARI del nuevo Pliego español RC-75 sólo lo podrán ser los P-550 y, en algún caso, algún P-450, pero en ningún caso $\longrightarrow$ en muy pocos- los P-350.

d) se ha dado, puede que un tanto maliciosamente, la interpretación de que el excesivo número real de categorías de cementos en el Pliego RC-75 puede repercutir en la "economía industrial" de las fábricas e inducir al "peligro de contaminación" de unos cementos con otros, al exigir mayor número de silos y no disponerse de los suficientes. Porque tal interpretación parte del supuesto no verosímil de que todas o la mayoría de las fábricas del país produzcan, simultáneamente o con alternancias de gran frecuencia, todos o la mayoría de los tipos, clases y categorías de cementos previstos en el Pliego RC-75. A este respecto cabe decir que cementos siderúrgicos y/o puzolánicos sólo podrán producirlos, en condiciones económicas rentables, aquellas fábricas lo suficientemente próximas a yacimientos de puzolanas naturales, o de centrales térmicas y plantas siderúrgicas que respectivamente produzcan cenizas volantes y escorias idóneas. Y, por otra parte, si bien es cierto que la utilización de adiciones activas tiene como finalidad inmediata, pero no única, ahorrar clínker, la finalidad económica real es la de ahorrar combustible y energía, en unas circunstancias críticas que lo exigen, y que muy probablemente tenderán a agravarse, exigiéndolo aún más en el futuro. Tampoco es despreciable la finalidad técnica de disponer de cementos más aptos para determinados empleos específicos relacionados con el calor de hidratación, la estabilidad y la durabilidad.

2) Al introducir para los cementos PA, S, PUZ y C el ensayo de expansión de las agujas Le Chatelier, en sustitución del ensayo de autoclave, fue una lástima no haber hecho extensiva dicha introducción y sustitución también en los cementos $\mathrm{P}$, con lo que todos hubieran quedado en condiciones equitativas y comparables, como sucede en la mayoría de los países; al tiempo que se unificaba así el ensayo de expansión, éste se hubiera simplificado, ahorrando equipo y tiempo a los laboratorios de control.

3) La antigua fórmula para establecer la puzolanicidad de un cemento a base de que el "índice puzolánico" (milimoles de $\mathrm{CaO} / \mathrm{litro}$ ) fuese menor que 360/(milimoles de alcalinidard total $-\mathrm{OH}^{-}-$litro) -15 , se ha sustituido, afortunadamente, por la situación del punto representativo del cemento en el gráfico de FRATINI (curva de solubilidad de la cal en presencia de álcalis y en función de la concentración de los mismos $-\mathrm{OH}^{-}-$).

Es decir, que se ha dado un paso atrás, que en este caso ha sido un paso adelante. La historia es curiosa: Al introducir en las normas españolas el ensayo de FRATINI, determinadas tendencias, tal vez exageradamente orientadas hacia los "divertimentos" de naturaleza matemática, intentaron y consiguieron sustituir la curva de FRATINI por "su" ecuación, así como expresar en función de ésta la condición de que un punto representativo de un cemento estuviese situado por debajo o por encima de dicha curva, según que el cemento fuese o no puzolánico. Se dio así prioridad a un formalismo matemático, olvidando que las curvas experimentales correspondientes a un fenómeno o a una condición química tienen un claro y preciso significado, y, por el contrario, no tienen porqué corresponder $-\mathrm{y}$ de hecho rara vez corresponden- a curvas teóricas expresables perfectamente por una ecuación. Tampoco son representables con absoluta fidelidad en todas sus zonas por ecuaciones deducidas de una forma más 
o menos sofisticada. Y así resultaba que si la ecuación establecida para la curva de FRATINI se ajustaba bien a ésta en el centro, se desajustaba en los extremos, o viceversa. Con lo cual, por un lado o por otro, cementos que no eran puzolánicos aparecían como tales, o al contrario. Esto daba —dió- lugar a polémicas.

4) Se ha debatido y sigue debatiéndose el tema de las adiciones "activas" (escorias, puzolanas y cenizas volantes) e "inertes". De las primeras se conocen -más o menoslos mecanismos y los efectos de su actividad. Pero en cuanto a las segundas, hay quienes, para bien o para mal, ponen en tela de juicio su "inercia", como la de todo material muy finamente molido, con una gran superficie específica "naciente", muy activa en ocasiones, y que desarrolla una gran energía superficial total. Argumentan que, por unos u otros mecanismos, estos materiales son capaces de dar lugar a reacciones de tipo topoquímico o epitáxico con los productos de la hidrólisis y/o de la hidratación del clínker y del cemento. Y consideran que, entre tales materiales, figuran, en efecto, las calizas y margas, tenidas en general y durante mucho tiempo por absolutamente "inertes".

Personalmente pienso que el dilucidar en qué aspectos, medidas y circunstancias, y con qué signo es activa una adición dada, es un problema del mayor interés, y soluble en cada caso únicamente por la vía experimental directa. Por otra parte, en general parece que cuando se habla de adiciones inertes no se quiere salvaguardar tanto su inactividad como su no nocividad (concepto que se recogía en el PCCH-61 y se mantenía en el PCCH-64). En tal caso el término "inerte" que el Diccionario define como "inactivo, ineficaz, estéril o inútil", puede y debe ser sustituido por el de "inocuo", esto es, "que no hace daño", cuando real y experimentalmente así sea.

5) Otro tema de controversia permanente es el de si los cementos que además de clínker y yeso contienen en distintas proporciones otros materiales -a los que los tranceses llaman "componentes secundarios" y los españoles llamamos "adiciones activas" en el Pliego RC-75- deben o no ostentar el apelativo de "portland". Las opiniones al respecto andan divididas.

El que les habla está de acuerdo en que en el "ámbito científico de la química del cemento", cemento portland es mezcla pulverulenta, en las debidas proporciones, obtenida por molturación conjunta hasta un grado adecuado de finura, de clínker portland y de regulador de fraguado - generalmente yeso (sulfato cálcico dihidrato)—.

Sin embargo, hay que reconocer que en el "ámbito técnico-comercial y en el de las normas" no ha sido ni es lo mismo. Las definiciones, y sobre todo las denominaciones y designaciones de los distintos tipos y clases de cemento son variables según los países, y aun a veces mutables con el tiempo, dentro de uno mismo. Así por ejemplo:

i) No siempre que un cemento contiene puzolana se llama -exclusivamente- cemento puzolánico. Se recuerda al respecto los esfuerzos tan denodados como infructuosos de los miembros italianos del Comité ISO TC-74 frente a miembros de otros países, para convencerles de que "cementos a la puzolana" y "cementos puzolánicos" no eran la misma cosa.

ii) Los cementos puzolánicos, tal como se entienden en general, se denominan de forma distinta, con o sin el apelativo de portland, según los países. De 17 países (100\%) que según el CEMBUREAU (5) tenían "cementos puzolánicos" normalizados en 1968 -incluida España-, 9* (53\%) los denominan cementos portland puzolánicos (o con puzolanas o de cenizas volcánicas, o de "trass"), pero siempre con el apellido portland; $7^{* *}(41 \%)$ de dichos países los denominan ce-

* Bulgaria - China - Holanda - Hungría - India - Méjico - Rumania - URSS - USA.

*:* República Federal de Alemania - Checoslovaquia - España - Italia - Japón - Portugal - Turquía. 
mentos puzolánicos (o con puzolana, o "a la puzolana", o de "trass"), pero sin el apellido portland; y un país* (6\%) los llama de las dos maneras, según se refiera a una de dos clases distintas incluidas en sus normas. Como se ve, las opiniones al respecto están equilibradamente repartidas, pero prevaleciendo ligeramente el criterio de aplicación del apelativo de portland.

iii) A pesar de ello, por lo que respecta a España el PCCH-64 contemplaba - y contempla - el cemento de tipo puzolánico PUZ (sin denominación de portland), y lo mismo sucede con el Pliego RC-75 respecto de los cementos PUZ-I y PUZ-II. Pero éste contempla además, y como novedad, los "cementos portland con adiciones activas", PA, una de las cuales puede ser una puzolana, tal y como ésta se define en dicho Pliego. Un cemento "portland con adición activa (puzolánica)" sería (es) equivalente a lo que se puede entender por cemento (portland) "a la puzolana" -véase i)-.

iv) En cuanto a los cementos siderúrgicos — con escorias de horno alto-, también se denominan de forma diferente — con y sin el apelativo de portland- según los países y la clase de cemento, dentro de su tipo. Así, de $25(100 \%)$ países que según el CEMBUREAU (5) los producían y normalizaban en 1968, incluida España, $8^{* *}$ de ellos $(32 \%$ ) los denominan cementos portland siderúrgicos (o férricos, o de hierro, o "ferroportland", o de escorias, o con escorias, o incluso de horno alto, pero siempre con el apelativo de portland); $4^{* * *}$ de ellos $(16 \%)$ los denominan cementos de horno alto (o de escorias, o con escorias, o metalúrgicos, pero sin el apelativo de portland); y $13^{* * * *}$ países (52\%) los llaman de las dos maneras, en función de distintas clases que las respectivas normas contemplan, según el contenido de escoria. Como es de apreciar, existe un cierto equilibrio de reparto, pero prevalece indudablemente el criterio de aplicación del apelativo de portland.

Por lo que afecta a España, el PCCH-64 incluía - e incluye- el cemento de tipo siderúrgico con denominación de portland, en sus dos clases: "portland siderúrgico" y "portland de horno alto", PS y PHA respectivamente, designaciones en las que la $\mathrm{P}$ tiene un claro y evidente significado. En cambio, y pese a lo anteriormente expuesto, en el Pliego RC-75 los cementos siderúrgicos (a secas), en las clases I, II y III, no ostentan el nombre de portland.

El Pliego RC-75 contempla además los "cementos portland con adiciones activas", PA, una de las cuales puede ser escoria, tal y como se define en el mismo. Un cemento "portland con adición activa (escoria)" sería - es- equivalente a lo que se puede entender por un cemento (portland) "a la escoria" - véase iii) y i) 一.

vi) Lo indicado en los puntos precedentes muestra a las claras que el paso del PCCH-64 al RC-75 mantiene o sitúa las denominaciones de los cementos puzolánicos y siderúrgicos en la línea de las preferencias de quien les habla, pese a que ello no corresponda - por ahora- a los usos y costumbres internacionales mayoritarios.

6) A propósito de los cementos puzolánicos PUZ y de los portland con adiciones activas PA cuando ésta es una puzolana, el Pliego RC-75 deja sin resolver una cuestión. A los cementos puzolánicos, para que lo sean, se les exige el cumplimiento de lo prescrito por el ensayo de FRATINI; a los portland con adición activa puzolánica, no. Por otra parte, a los primeros no se les fija límite superior de contenido de puzola-

\footnotetext{
* Yugoslavia.

-5*:* Africa del Sur - Bulgaria - China - España - Hungria - India - Inglaterra - Japón.

***:* Italia - Polonia - Suecia - Turquia.

*:*** República Democrática Alemana - República Fecieral de Alemania - Austria - Bélgica - Checoslovaquia - Finlandia - Francia - Holanda - Portugal - Rumania - URSS - USA - Yugoslavia.
} 
na, quedando fijado éste por el extremo inferior en 20 por ciento; a los segundos se les impone este mismo valor como límite superior. $Y$ ahora surge el problema: dado que existen puzolanas muy activas que en dosis inferior al $20 \%$ y molidas con determinados clínkeres a finura normal o algo superior son capaces de dar cementos que cumplen con el ensayo de FRATINI, ¿qué pasa con estos cementos: son portland de adición (con puzolana), o son puzolánicos? Indudablemente son las dos cosas, pero a la hora de clasificarlos y encasillarlos surgirá la duda, pues no faltarán opiniones para preferir su encuadramiento de una forma o de otra. En realidad, a mi juicio tales cementos deberían ser considerados, sobre todo, como puzolánicos, pues ante el hecho de cumplir con el ensayo de FRATINI, la proporción de puzolana que contengan (mayor o menor del $20 \%$ ) es aspecto secundario.

7) Tema no menos polémico, es el que surge a propósito de los cementos con propiedades adicionales que, siempre según el Pliego RC-75, son los de alta resistencia inicial (ARI), los "portland" resistentes al yeso (Y), los de bajo calor de hidratación (BC) y los blancos (B). Todos ellos portland $\mathrm{P}$, y con la posibilidad de ser también considerados blancos únicamente algunos cementos compuestos C. Acerca de esto habría tanto que decir que alargaría demasiado estas consideraciones

Sin embargo, vaya aquí un solo comentario: hay otros cementos distintos de los incluidos en el Pliego RC-75 como poseedores de propiedades adicionales, que tienen per se (es decir, por su naturaleza) algunas de las propiedades adicionales destacadas en el mismo (e incluso otras que no se destacan) y en mayor cuantía que los portland. A mi entender, ante este hecho no cabe salir del paso, como se hace en el Pliego RC-75, indicando que "la normalización de las propiedades adicionales en el cemento de tipo portland $-\mathrm{y}$ en el cemento de tipo compuesto, habría que añadirno implica que no puedan poseer las mismas propiedades cementos de otros tipos". Las pueden poseer y de hecho y sin duda las poseen y las tienen bien reconocidas; esas y otras distintas que, en cambio, no poseen $\_0$ no poseen en la misma medida, porque no pueden poseerlas - los cementos de tipo portland o compuesto. Así sucede, por ejemplo, con la resistencia química frente al agua de mar (España tiene mucho litoral y en él y cerca de él se hacen, cada vez más, muchas obras de todo tipo). Repito que el comentario sobre el tema podría ser muy amplio y variado, ya que el Pliego RC-75, desde el punto de vista de la durabilidad frente al ataque químico, sólo considera cementos portland resistentes al yeso, cuando ni son los únicos resistentes, ni el yeso es el único medio agresivo, aunque en España tenga gran importancia. A mayor abundamiento, los cementos portland resistentes al yeso no son precisamente los más resistentes frente a otros sulfatos, como el magnésico, por ejemplo, ni frente al agua de mar y otros medios de naturaleza ácida.

8) Y ahora, echemos una ojeada rápida a las especificaciones de tipo químico.

Por lo que respecta a la pérdida al fuego y al residuo insoluble de los cementos, en el PCCH-64 ambos eran iguales para los portland y siderúrgicos - y muy aproximadamente iguales a las medias de todos los países que en el mundo fijan valores máximos admisibles para ambos conceptos analíticos (5)_. En el Pliego RC-75 estos conceptos tienen limitaciones distintas en el caso de los cementos portland, en función de la categoría resistente, siendo más estrictas aquéllas, cuanto mayor es ésta. Para establecerlo así se ha tenido posiblemente en cuenta el hecho de que los cementos portland "puros" constan sólo de clínker y yeso. Pero ni uno ni otro son productos químicos puros, ni una fábrica de cemento es una planta de fármacos. Así, pues, el clínker puede tener una cierta proporción (por insignificante que sea) de materia insoluble; a igualdad de todo lo demás, tanto menor cuanto mejor cocido esté. La clinkerización debe ser tanto más completa cuanto mayor sea el grado de saturación del crudo o, si prefiere, cuanto mayor contenido de silicato tricálcico se pretenda 
que tenga el clínker. $Y$ es tanto más completa cuanto menor es el contenido de cal libre y/o de residuo insoluble. $\mathrm{Y}$ dado que en ninguna norma se especifica sobre cal libre, una garantía de buena cocción en el caso de clínkeres de gran saturación, aptos para cementos de alta categoría resistente, puede ser el fijar para éstos unos límites de residuo insoluble más restrictivos que para los de categoría resistente más baja.

Por otra parte, las impurezas del yeso pueden ser de naturaleza arcillosa y/o margocaliza, y aportar, por lo tanto, residuo insoluble y/o pérdida al fuego al cemento (aparte de la pérdida al fuego aportada por el propio yeso puro, con su agua de cristalización). A igualdad de peso, la caliza pura aporta en concepto del dióxido de carbono una pérdida al fuego aproximadamente doble que la que aporta el yeso puro en concepto de agua de cristalización; lo cual quiere decir que cuanto mayor impureza caliza (o margosa) contiene un yeso, mayor pérdida al fuego aporta al cemento. Y como, en buena lógica, los yesos más puros (como antes los clínkeres más saturados y mejor cocidos) suelen ser empleados en los cementos de mayor categoría resistente, para éstos se puede especificar también una pérdida al fuego menor (o, de otro modo, una pérdida al fuego mayor para el cemento de categoría resistente más baja).

Claro está, y con esto me anticipo a posibles y obligadas observaciones, que no se puede olvidar el hecho real y práctico de que, por lo general, cada fábrica utiliza una sola y misma clase de yeso para todos los cementos que produce. Por lo tanto, hubiera sido más realista y comprensible el haber mantenido para todos los cementos P, PA y S del Pliego RC-75 las mismas especificaciones del PCCH-64 a saber: $4 \%$ máximo para la pérdida al fuego y $3 \%$ máximo para el residuo insoluble, dado que, como se dijo al principio, tales valores son muy aproximadamente la media de los especificados por todos los países con normas para cemento.

\section{FUTURO}

Por el Decreto de la Presidencia del Gobierno (español) en el que se aprueba el Pliego RC-75 y se dispone su entrada en vigor a partir del día 1 de junio de 1976, se crea la Comisión Permanente encargada de la revisión de dicho Pliego y de la propuesta y realización de trabajos relacionados con el mismo.

Desde que el Pliego RC-75 se ha hecho público, han ido llegando al Presidente de la Comisión Permanente escritos con observaciones hechas por parte de personas, bien a título privado, o bien como representantes de industrias cementeras, del hormigón, de la construcción y de laboratorios.

Las observaciones se refieren, según los casos, a los valores especificados, a los tipos, clases y categorías de los cementos, a sus propiedades adicionales, o a los métodos de análisis y ensayos.

Todas ellas deberán ser tenidas en cuenta con carácter permanente por la Comisión.

Por otra parte, se entiende que ésta deberá estar, ahora más que nunca, al tanto de las tendencias y orientaciones que en materia de normalización de cemento se marquen en las organizaciones internacionales, principalmente en la ISO y en el CEN (Comité Europeo de Normalización), pues este último no va a limitar su radio de acción a los actuales nueve países del Mercado Común y asociados, sino que va a trascender, cuando menos, a los restantes países europeos occidentales. 
El Pliego RC-75 no sólo no es perfecto, sino que dista mucho de serlo, y a tenor de lo que precede tendrá que ir perfeccionándose, de acuerdo con las sugerencias de las partes interesadas, encauzadas a través de la Comisión Permanente.

Materia no falta, puesto que ya hay aspectos nuevos que considerar, aparte de otros viejos que se vuelven a replantear. Por ejemplo, el del ensayo de los cementos (en pasta y mortero) con factor agua/cemento fijo $-\mathrm{y}$ por consiguiente a consistencia variable-, o a consistencia fija y con factor agua/cemento variable, en relación con la finura de los cementos, con las resistencias de los mismos y con el empleo del cemento en hormigón. Este problema se puede presentar así:

a) a igualdad de consistencia un mismo cemento molido a dos finuras distintas -0 dos cementos distintos y con finuras diferentes- exigen también diferente cantidad de agua: mayor los más finos y menor los menos finos, como es evidente. Pero no se podrá decir a priori en cuál de los dos casos se obtendrán mayores resistencias, puesto que depende, en cada uno de ellos, de la influencia relativa del factor agua/cemento por una parte, y de la finura del cemento, por otra. Pero no sólo de la finura determinada y expresada como superficie específica BLAINE, sino principalmente de la curva granulométrica del cemento en cada caso, ya que la superficie específica no es más que un solo valor numérico pretendidamente representativo de toda una gama, secuencia o transición, más o menos continua, de tamaños de partículas de un material pulverulento, es decir, de su granulometría. Y podría darse la circunstancia de que un cemento de finura BLAINE mayor diena resistencias más bajas que otro de finura BLAINE menor;

b) a igualdad de agua -o de relación a/c_ el cemento más fino dará una consistencia más plástica o seca y el menos fino la dará más fluida o húmeda. En esta situación sí se podrá decir que, en la generalidad de los casos, el cemento más fino dará resistencias más altas que el menos fino, como por otra parte es lógico, y a ello estamos acostumbrados.

El amasado mecánico de la pasta para los ensayos de consistencia y fraguado de los cementos (tendencia del CEN - GT 51, GE 2-) para evitar errores debidos al factor personal de los operadores; la definición y determinación del principio y del fin del fraguado; las arenas normalizadas para los ensayos de resistencia en mortero y la influencia de su fracción más fina; éstos y tantos otros son problemas, ya planteados algunos, que la Comisión Permanente del Pliego RC-75, con las colaboraciones necesarias, tendrá que ir estudiando y resolviendo, lo más de acuerdo posible con los organismos internacionales de normalización.

Finalmente, a efectos interiores del país, y siguiendo una tradición ya establecida en los anteriores cambios de normas, el IETCC proyecta y ya tiene en fase bastante avanzada de realización, la publicación de un Manual que vendrá a ser una especie de "Código Práctico del Empleo de los Cementos del RC-75". En él se señalarán, a título orientativo, los distintos empleos específicos (así como las observaciones pertinentes sobre los mismos) y las contraindicaciones de uso de los diferentes tipos, clases y categorías de cementos del Pliego RC-75.

En dicho manual, y en su parte explicativa, se procurará aclarar puntos oscuros y suplir, al menos en parte, algunas insuficiencias del Pliego RC-75, en espera de que, en su momento, sea la Comisión Permanente la que subsane cuanto en el mismo sea de subsanar.

Señores: He pretendido dar una panorámica de la evolución de las normas españolas para cemento en el tiempo. He procurado describir y comentar algunos de los puntos más destacados de las nuevas normas que entrarán en vigor a partir del próximo mes de ju- 
nio. No me han dolido prendas a la hora de emitir juicios críticos y de manifestar reservas o disconformidades con determinados aspectos parciales de las mismas, que ciertamente no serán tal vez los únicos criticables.

En todo caso estoy a su disposición para desarrollar con ustedes un coloquio sobre lo que deseen comentar, aclarar o puntualizar.

C UADRO 1

Clasificación de los cementos

\begin{tabular}{|c|c|c|c|}
\hline Tipos & Clases & Categorías & Designaciones \\
\hline Portland & & $\begin{array}{l}350 \\
450 \\
550\end{array}$ & $\begin{array}{l}P-350 \\
P-450 \\
P-550\end{array}$ \\
\hline Portland con adiciones activas & & $\begin{array}{l}350 \\
450 \\
550\end{array}$ & $\begin{array}{l}\text { PA-350 } \\
\text { PA-450 } \\
\text { PA-550 }\end{array}$ \\
\hline \multirow{3}{*}{ Siderúrgico } & 1 & $\begin{array}{l}350 \\
450\end{array}$ & $\begin{array}{l}\text { S-I-350 } \\
\text { S-I-450 }\end{array}$ \\
\hline & II & 350 & S-11-350 \\
\hline & III & $\begin{array}{l}250 \\
350\end{array}$ & $\begin{array}{l}\text { S-III-250 } \\
\text { S-III-350 }\end{array}$ \\
\hline \multirow{2}{*}{ Puzolánico } & 1 & $\begin{array}{l}250 \\
350 \\
450\end{array}$ & $\begin{array}{l}\text { PUZ-I-250 } \\
\text { PUZ-I-350 } \\
\text { PUZ-1-450 }\end{array}$ \\
\hline & II & $\begin{array}{l}250 \\
350 \\
450\end{array}$ & $\begin{array}{l}\text { PUZ-II-250 } \\
\text { PUZ-II-350 } \\
\text { PUZ-II-450 }\end{array}$ \\
\hline Compuesto & & 200 & $C-200$ \\
\hline Aluminoso & & 550 & A-550 \\
\hline \multirow[t]{2}{*}{ Natural } & Lento & $\begin{array}{l}30 \\
80\end{array}$ & $\begin{array}{l}\text { NLL-30 } \\
\text { NL-80 }\end{array}$ \\
\hline & Rápido & 20. & NR-20 \\
\hline
\end{tabular}

C UADRO 2

Cementos con propiedades adicionales

\begin{tabular}{|c|c|c|c|c|}
\hline \multirow{2}{*}{ Tipo } & $\begin{array}{c}\text { Cementos de alta } \\
\text { resistencia inicial }\end{array}$ & $\begin{array}{c}\text { Cementos portland } \\
\text { resistentes al yeso }\end{array}$ & $\begin{array}{c}\text { Cementos de bajo } \\
\text { calor de hidratación }\end{array}$ & Cementos blancos \\
\hline & \multicolumn{2}{|c|}{ Clases y Categorías } \\
\hline \multirow{3}{*}{ Portland } & P-350-ARI & P-350-Y & & P-350-B \\
& P-450-ARI & P-450-Y & P-350-BC & $\begin{array}{c}\text { P-450-B } \\
\text { P-550-ARI }\end{array}$ \\
\hline Compuesto & P-550-Y & & C-200-B \\
\hline
\end{tabular}


C UADRO 3

Prescripciones relativas a las características quimicas

\begin{tabular}{|c|c|c|c|c|c|c|c|c|c|c|c|c|c|c|c|c|c|c|c|c|c|c|}
\hline TIPO & & ORTLAI & & $\begin{array}{r}\text { POR } \\
\text { ADICIO }\end{array}$ & $\begin{array}{l}\text { TLAND } \\
\text { NES AC }\end{array}$ & $\begin{array}{l}\text { CON } \\
\text { TIVAS }\end{array}$ & & SIDEI & RURG & & & & & UZOL & NICO & & & 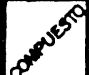 & & & ATURAL & \\
\hline CLASE & & & & & & & & I & III & & & & I & & & III & & & & LEN & ITO & RAPIDO \\
\hline CATEGORIA & 350 & 450 & 550 & 350 & 450 & 550 & 350 & 450 & 350 & 250 & 350 & 250 & 350 & 450 & 250 & 350 & 450 & 200 & 550 & 30 & 80 & 20 \\
\hline DESIGNACION & P.350 & P.450 & P.550 & PA.350 & $P A-450$ & PA.550 & $s \cdot 1 \cdot 350$ & $S \cdot I-450$ & $\begin{array}{l}5 \cdot \pi \\
350 \\
\end{array}$ & $\begin{array}{l}\text { S-II } \\
250\end{array}$ & $\begin{array}{l}\text { S-III } \\
350 \\
\end{array}$ & $\begin{array}{l}\text { PUZ } \\
I-250\end{array}$ & $\begin{array}{l}\text { PUZ } \\
I-350 \\
\end{array}$ & $\begin{array}{l}P U Z \\
I-450 \\
\end{array}$ & $\begin{array}{l}\text { PUZ } \\
\text { II- } 250\end{array}$ & \begin{tabular}{|r|} 
PUZ \\
-350 \\
\end{tabular} & $\begin{array}{l}\text { PUZ } \\
I-450 \\
\end{array}$ & $c-200$ & $A-550$ & $N L-30$ & NL-80 & NR-20 \\
\hline $\begin{array}{l}\text { PEROIDA AL FUEGO } \\
\text { MAX. } \%\end{array}$ & 4 & 3.5 & 3.5 & 4 & 4 & 4 & 4 & 4 & 4 & 4 & 4 & & & & & & & & & & & \\
\hline $\begin{array}{l}\text { RESIDUO INSOLUBLE } \\
\text { MAX. \% }\end{array}$ & 3 & 2.5 & 2.5 & $4^{*}$ & $4^{*}$ & $4^{*}$ & 3 & 3 & 3 & 3 & 3 & & & & & & & & & & & \\
\hline $\begin{array}{c}\text { OXIDO MAGNESICO } \\
\text { MgO } \\
\text { MAX. } \%\end{array}$ & 5 & 5 & 5 & 5 & 5 & 5 & & & & & & & & & & & & & & & & \\
\hline $\begin{array}{c}\text { TRIOXIDO DE AZUFRE } \\
\text { SO }_{3} \\
\text { MAX. } \%\end{array}$ & 4 & 4.5 & 4.5 & 4 & 4 & 4 & 4.5 & 4.5 & 4.5 & 4.5 & 4.5 & 3.5 & 3.5 & 3.5 & 3.5 & 3.5 & 3.5 & 3.5 & & & & \\
\hline $\begin{array}{c}\text { OXIDO ALUMINICO } \\
\mathrm{Al}_{2} \mathrm{O}_{3} \\
\mathrm{MIN.} \%\end{array}$ & & & & & & & & & & & & & & & & & & & 36 & & & \\
\hline $\begin{array}{c}\text { SULFUROS } \\
\text { S2 } \\
\text { MAX.\% }\end{array}$ & & & & & & & & & & & & & & & & & & & 0.1 & & & \\
\hline INDICE PUZOLANICO & & & & & & & & & & & & $\begin{array}{l}\text { CUMP } \\
\text { ALA } \\
\text { RIOE: }\end{array}$ & $\begin{array}{l}\text { IRAN } \\
\text { UZOLA } \\
\text { TABLE }\end{array}$ & $\begin{array}{l}\text { A PRE } \\
\text { NICIDA } \\
\text { IDO PC }\end{array}$ & $\begin{array}{l}\text { SCRIPCI } \\
\text { PSEGU } \\
\text { R LA F }\end{array}$ & $\begin{array}{l}\text { ON REL } \\
\text { N ELC } \\
\text { IG.8.21 }\end{array}$ & $\begin{array}{l}\text { ATIVA } \\
\text { RITE- }\end{array}$ & & & & & \\
\hline $\begin{array}{c}\text { MATERIA INERTE } \\
\text { MAX \% }\end{array}$ & & & & & & & & & & & & & & & & & & 35 & & & & \\
\hline
\end{tabular}

* Si la adicion es de puzolana el limite se eleva al $8 \%$ y si es de ceniza volante no se limita, pero en todo caso siempre se determinara el limite de RESIDUO INSOLUBLE. TANTO EN UN CASO COMO EN EL OTRO, CUANDO EL VALOR SEA SUPERIOR AL 4\%, EL FABRICANTE ESTARA OBLIGADO A SUMINISTRAR MUESTRAS DEL 


\section{U A D R O 4}

Prescripciones relativas a las características físicas y mecánicas

\begin{tabular}{|c|c|c|c|c|c|c|c|c|c|c|c|c|c|c|c|c|c|c|c|c|c|c|}
\hline \multirow{3}{*}{$\frac{\text { TIPO }}{\text { CLASE }}$} & \multicolumn{3}{|c|}{ PORTLAND } & \multicolumn{3}{|c|}{$\begin{array}{l}\text { PORTLAND CON } \\
\text { ADICIONES ACTIVAS }\end{array}$} & \multicolumn{5}{|c|}{ SIDERURGICO } & \multicolumn{6}{|c|}{ PUZOLANICO } & & & \multicolumn{3}{|c|}{ NATURAL } \\
\hline & & & & & & & \multicolumn{2}{|c|}{$I$} & \multirow{2}{*}{$\frac{\text { II }}{350}$} & \multicolumn{2}{|c|}{ III } & \multicolumn{3}{|c|}{ PUZOLANA EN GENERAL } & \multicolumn{3}{|c|}{ DE CENIZA VOLANTE } & \multirow[b]{2}{*}{200} & \multirow[b]{2}{*}{550} & \multicolumn{2}{|c|}{ LENTO } & \multirow{2}{*}{$\frac{\text { RAPIDO }}{20}$} \\
\hline & 350 & 450 & 550 & 350 & 450 & 550 & 350 & 450 & & 250 & 350 & 250 & 350 & 450 & 250 & 350 & 450 & & & 30 & 80 & \\
\hline DESIGNACION & $P-350$ & $P .450$ & P-550 & PA. 350 & PA.450 & PA.550 & $5 \cdot 1 \cdot 350$ & S.I.450 & $\begin{array}{l}5 \cdot \pi \\
350 \\
\end{array}$ & $\begin{array}{l}S-m \\
250\end{array}$ & $\begin{array}{l}\text { S-m } \\
350\end{array}$ & $\begin{array}{l}\text { PUZ } \\
I-250 \\
\end{array}$ & $0 \begin{array}{r}P U Z \\
-350\end{array}$ & $\begin{array}{r}P U Z \\
I-450\end{array}$ & $\begin{array}{l}\text { PUZ } \\
\text { II-250 } \\
\end{array}$ & $\begin{array}{r}P U Z \\
I I-350 \\
\end{array}$ & $\begin{array}{l}\text { PUZ } \\
\text { III-450 } \\
\end{array}$ & C-200 & $A-550$ & $N L-30$ & $N L-80$ & NR-20 \\
\hline $\begin{array}{l}\text { FINURA DE MOLIDO } \\
\text { POR RESIDUO MAX.\% EN } \\
\text { TAMIZ DE } 4,900 \text { mallos } / \mathrm{cm}^{2}\end{array}$ & 15 & 15 & 15 & 15 & 15 & 15 & 15 & 15 & 15 & 15 & 15 & 15 & 15 & 15 & 15 & 15 & 15 & 15 & 15 & 15 & 15 & 15 \\
\hline $\begin{array}{l}\text { TIEMPOS DE FRAGUADO } \\
\text { PRINCIPIO, DESPUES DE } \\
\text { FINAL, ANTES DE }\end{array}$ & $\begin{array}{l}45 \mathrm{~min} \\
12 \mathrm{hr}\end{array}$ & $\begin{array}{l}45 \mathrm{~min} \\
12 \mathrm{hr}\end{array}$ & $\begin{array}{l}45 \mathrm{~min} \\
12 \mathrm{hr}\end{array}$ & $\begin{array}{l}45 \mathrm{~min} \\
12 \mathrm{hr}\end{array}$ & $\begin{array}{c}45 \mathrm{~min} \\
12 \mathrm{hr}\end{array}$ & $\begin{array}{l}45 \mathrm{~min} \\
12 \mathrm{hr}\end{array}$ & $\begin{array}{c}45 \mathrm{~min} \\
12 \mathrm{hr}\end{array}$ & $\begin{array}{l}45 \mathrm{~min} \\
12 \mathrm{hr}\end{array}$ & $\begin{array}{l}45 \mathrm{~min} \\
12 \mathrm{hr}\end{array}$ & $\begin{array}{l}45 \mathrm{~min} \\
12 \mathrm{hr}\end{array}$ & $\begin{array}{c}45 \mathrm{~min} \\
12 \mathrm{hr}\end{array}$ & $\begin{array}{c}45 \mathrm{~min} \\
12 \mathrm{hr}\end{array}$ & $\begin{array}{c}45 \mathrm{~min} \\
12 \mathrm{hr}\end{array}$ & $\begin{array}{l}45 \mathrm{~min} \\
12 \mathrm{hr}\end{array}$ & $\begin{array}{l}45 \mathrm{~min} \\
12 \mathrm{hr}\end{array}$ & $\begin{array}{l}45 \mathrm{~min} \\
12 \mathrm{hr}\end{array}$ & $\begin{array}{l}45 \min \\
12 \mathrm{hr}\end{array}$ & $\begin{array}{l}45 \mathrm{~min} \\
12 \mathrm{hr}\end{array}$ & $\begin{array}{l}30 \mathrm{~min} \\
12 \mathrm{hr}\end{array}$ & $\begin{array}{l}30 \mathrm{~min} \\
12 \mathrm{hr}\end{array}$ & $\begin{array}{l}30 \mathrm{~min} \\
12 \mathrm{hr}\end{array}$ & $\begin{array}{l}2 \min \\
30 \min \end{array}$ \\
\hline $\begin{array}{l}\text { EXPANSION EN } \\
\text { AUTOCLAVE } \\
\text { MAXIMA EN \% }\end{array}$ & 1 & 1 & 1 & & & & & & & & & & & & & & & & & & & \\
\hline $\begin{array}{l}\text { EXPANSION POR } \\
\text { AGUJAS } \\
\text { MAXIMA EN } \mathrm{mm} .\end{array}$ & & & & 10 & 10 & 10 & 10 & 10. & 10 & 10 & 10 & 10 & 10 & 10 & 10 & 10 & 10 & 10 & & & & \\
\hline $\begin{array}{r}\text { RESISTENCIA EN KG/cm² } \\
\text { MINIMA FLEXOTRACCIONA } \\
\text { LAS EDADES DE } 1 \text { DIA } \\
3 \text { DIAS } \\
7 \text { DIAS } \\
28 \text { DIAS } \\
\text { MINIMA A COMPRESION A } \\
\text { LAS EDADES DE } 1 \text { DIA } \\
3 \text { DIAS } \\
7 \text { DIAS } \\
28 \text { DIAS }\end{array}$ & $\begin{array}{l}175 * \\
250 \\
350\end{array}$ & $\begin{array}{l}250 \\
350 \\
450\end{array}$ & $\begin{array}{l}350 \\
450 \\
550\end{array}$ & $\begin{array}{l}40 * \\
50 \\
60\end{array}$ & $\begin{array}{l}250 \\
350 \\
450\end{array}$ & $\begin{array}{l}350 \\
450 \\
550\end{array}$ & $\begin{array}{l}40 * \\
50 \\
60\end{array}$ & $\begin{array}{l}250 \\
350 \\
450\end{array}$ & $\begin{array}{l}175 * \\
250 \\
350\end{array}$ & $\begin{array}{l}175 \\
250\end{array}$ & $\begin{array}{l}175 * \\
250 \\
350\end{array}$ & $\begin{array}{l}175 \\
250\end{array}$ & $\begin{array}{l}40 * \\
50 \\
60\end{array}$ & $\begin{array}{l}250 \\
350 \\
450\end{array}$ & $\begin{array}{l}175 \\
250\end{array}$ & $\begin{array}{l}40 * \\
50 \\
60\end{array}$ & $\begin{array}{l}250 \\
350 \\
450\end{array}$ & $\begin{array}{l}100 \\
200\end{array}$ & $\begin{array}{c}65 \\
80 \\
450 \\
550\end{array}$ & $\begin{array}{l}20 \\
30\end{array}$ & $\begin{array}{l}15 \\
25\end{array}$ & $\begin{array}{l}15 \\
20\end{array}$ \\
\hline
\end{tabular}

* este valor tiene caracter indicativo. no es motivo de rechazo no alcanzar este valor si se cumplen las resistencias A 7 Y 28 DIAS INDICADAS. 
C U A D R 5

Prescripciones relativas a los cementos con propiedades adicionales

\begin{tabular}{|c|c|c|c|c|}
\hline DENOMINACION & $\begin{array}{l}\text { CEMENTOS DE ALTAS } \\
\text { RESISTENCIAS INICIALES }\end{array}$ & $\begin{array}{l}\text { CEMENTOS PORTLAND } \\
\text { RESISTENTES AL YESO }\end{array}$ & $\begin{array}{l}\text { CEMENTOS DE BAJA } \\
\text { CALOR DE HIDRATACION }\end{array}$ & CEMENTOS BLANCOS \\
\hline DESIGNACION ADICIONAL & $A R I$ & 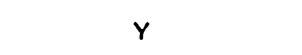 & $B C$ & B \\
\hline $\begin{array}{l}\text { COMPOSICION QUIMICA } \\
\text { CONTENIDOS MAXIMOS } \\
\text { EN \% DE C } C_{3} A \\
C_{4} A F+C_{3} A\end{array}$ & & $\begin{array}{c}5 \\
22\end{array}$ & & \\
\hline $\begin{array}{l}\text { TIEMPO DE FRAGUADO } \\
\text { PRINCIPIO, DESPUES DE } \\
\text { FINAL, ANTES DE }\end{array}$ & $\begin{array}{l}30 \mathrm{~min} \\
12 \mathrm{hr}\end{array}$ & & & \\
\hline $\begin{array}{l}\text { RESISTENCIA EN } \mathrm{KP} / \mathrm{cm}^{2} \\
\text { MINIMA A COMPRESION } \\
\text { A LA EDAD DE. } 48 \text { horos }\end{array}$ & 250 & & & \\
\hline $\begin{array}{c}\text { CALOR DE HIDRATACION } \\
\text { MAXIMO EN CALORIAS / gramo } \\
\text { A } 7 \text { dias } \\
\text { A } 28 \text { dios }\end{array}$ & & & $\begin{array}{l}65 \\
75\end{array}$ & \\
\hline
\end{tabular}


R E F E R E N C I A S

(1) "Pliego General de Condiciones para la Recepción de Aglomerantes Hidráulicos en Obras de Carácter Ofical". Curso para la Formación de Auxiliares de Laboratorio en la Industria del Cemento. Vol. III. IETCC, Madrid, 1954.

(2) PCCH-61: "Pliego General de Condiciones para la Recepción de Conglomerantes Hidráulicos en las Obras de Carácter Oficial". Normas y Manuales del IETCC. Madrid 1961.

(3) PCCH-64: "Pliego General de Condiciones para la Recepción de Conglomerantes Hidráulicos en las Obras de Cáracter Oficial". Normas y Manuales del IETCC. Madrid, 1964.

(4) RC-75: "Pliego de Prescripciones Técnicas Generales para la Recepción de Cementos 1975". Servicio de Publicaciones de la Secretaría General Técnica del Ministerio de Obras Públicas. Madrid, 1975.

(5) CEMBUREAU: "Cement Standards of the World: Portland Cement and its Derivatives". CEMBUREAU, París, 1968. 
